

\section{DISCLAIMER}

This document was prepared as an account of work sporsored by an agency of the United States Government. Neither the United States Government nor the University of California nor any of their employees, makes any warranty, express or implied, or assumes any legal liability or responsibility for the accuracy, completeness, or usefuluess of any information, apparatus, product, or process disclosed, or represents that its use would not infringe privately owned rights. Reference herein to any specific commercial products process, or service by trade name, trademark, manufacturer, or otherwise, does not necessarily constitute or imply its endorsement, recommendation, or favoring by the United States Government or the University of California. The views and opinions of authors expressed herein do not necessarily state or reflect those of the United States Government or the University of California, and shall not be used for advertising or product endorsement purposes.

This report has been reproduced directly from the best available copy.

Available to DOE and DOE contractors from the Ofice of Scientific and Technical Information

P.O. Box 62, Oak Ridge, TN 37831

Prices available from (615) 576-8401, FTS 626-8401

Available to the public from the

National Technical Information Service

U.S. Depariment of Commerce

5285 Port Royal Rd.

Springtied, VA 2216 


\title{
INSENS Classification Algorithm Report
}

\author{
Jose E. Hernandez, Christopher J. Frerking, and Dave W. Myers
}

\author{
Lawrence Livermore National Laboratory \\ POBox 808 L-156 \\ Livermore, CA 94550
}

This report describes a new algorithm developed for the Imigration and Naturalization Service (INS) in suppori of the INSENS project for classifying vehicles and pedestrians using seismic data. This algorithm is less sensitive to nuisance alarms due to environmental events than the previous algorithm. Furthermore, the algorithm is simple enough that it can be implemented in the 8-bit microprocessor used in the INSENS system.

\section{INTRODUCTION}

The Immigration and Naturalization Services (INS) agency is currently funding a project to improve the performance of existing algorithms for detecting vehicles and pedestrians illegally crossing the United States land borders. A portable, battery-powered, system has been designed consisting of signal processing electronics, an RF transmitter, and an 8-bit microprocessor for processing and classifying events measured from external transducers. Once an event is detected and classified as a target event, a signal is transmitted to the local border patrol station to indicate a possible illegal border crossing activity near the sensor location. The sensors currently in use include passive infra-red (IR) sensors, magnetometers, and geophones. This report describes a new algorithm to be incorporated into the INSENS system for detecting and classifying vehicles and pedestrians using seismic data collected from the geophone sensor. This algorithm is less sensitive to nuisance alarms $d$ than the previous algorithm. Furthermore, its computational requirements are not too demanding, and therefore very suitable for the INSENS microprocessor.

\section{TARGET SIGNATURE STUDIES}

\section{Target/Non-Target Definition}

After several interviews with members of each of the four participating border patrol sectors, it was decided that the main targets of interest would consist of vehicles and pedestrians. We also identified a set of typical sources responsible for causing nuisance alarms. A list of these sources has been included in Appendix A (page 10). After evaluating all the comments collected from the different sectors it was decided that we would focus our efforts in improving the classification algorithm for the geophones, since many of the problems associated with geophones could potentially be solved in software using the existing processing hardware.

\section{Data Collection}

Most of the data used for our algorithm development was collected locally from several sites in the Livermore area. Some additional data from the southern border was also provided to us by Sam Campbell and Louis Larocque. Table 1 shows the total amount of useful data that was 
collected for this project, organized by category. A prototypical signature for each category is depicted in Figure 1.

\begin{tabular}{|c|c|}
\hline Category & Samples \\
\hline \hline person & 26 \\
\hline people (>1) & 42 \\
\hline vehicle & 50 \\
\hline bicycle & 3 \\
\hline creek & 14 \\
\hline background & 29 \\
\hline train & 3 \\
\hline $\begin{array}{c}\text { irrigation } \\
\text { canal }\end{array}$ & 6 \\
\hline
\end{tabular}

Table 1: Collected data by category for the new algorithm.

\section{Data Reduction}

In order to prototype the pattern recognition algorithms, the data had to be organized by category into a database. Some of the signals also had to be edited to remove artifacts created by the data gathering process that would not occur during normal use. An interactive graphical user interface (GUI) was developed to facilitate this task. A picture of the GUI tool is shown in Figure 2. By simply clicking buttons, the user can march through a whole data set previously stored on disk, and plot, edit, and save the waveforms into the proper database directory. Note that the data for the categories creek, train, and irrigation canal were all stored under the category other. Furthermore, records that exhibited very bad artifacts were saved under the categories of difficult or bad depending on how bad the artifacts were.

\section{ALGORITHM DEVELOPMENT}

\section{Current Algorithm Overview}

The initial algorithm included in the engineering models for processing the seismic data obtained from the geophone sensor calculates the ratio of the area under the waveform divided by the area under the envelope of the waveform. If this ratio is above a predefined threshold level (about 0.5 ), the event is classified as a vehicle, otherwise it is classified as pedestrian. Note that once an event is detected by the analog circuitry in the system, the event is always classified, unless it is a very short event (less than 2 secs). This is the reason why certain events such as creeks, irrigation canals, heavy rain, and trains tend to be erroneously reported as target events. We have developed a new algorithm for discriminating pedestrians and vehicles from other events. Furthermore, the current algorithm for discriminating pedestrians from vehicles has been improved. 

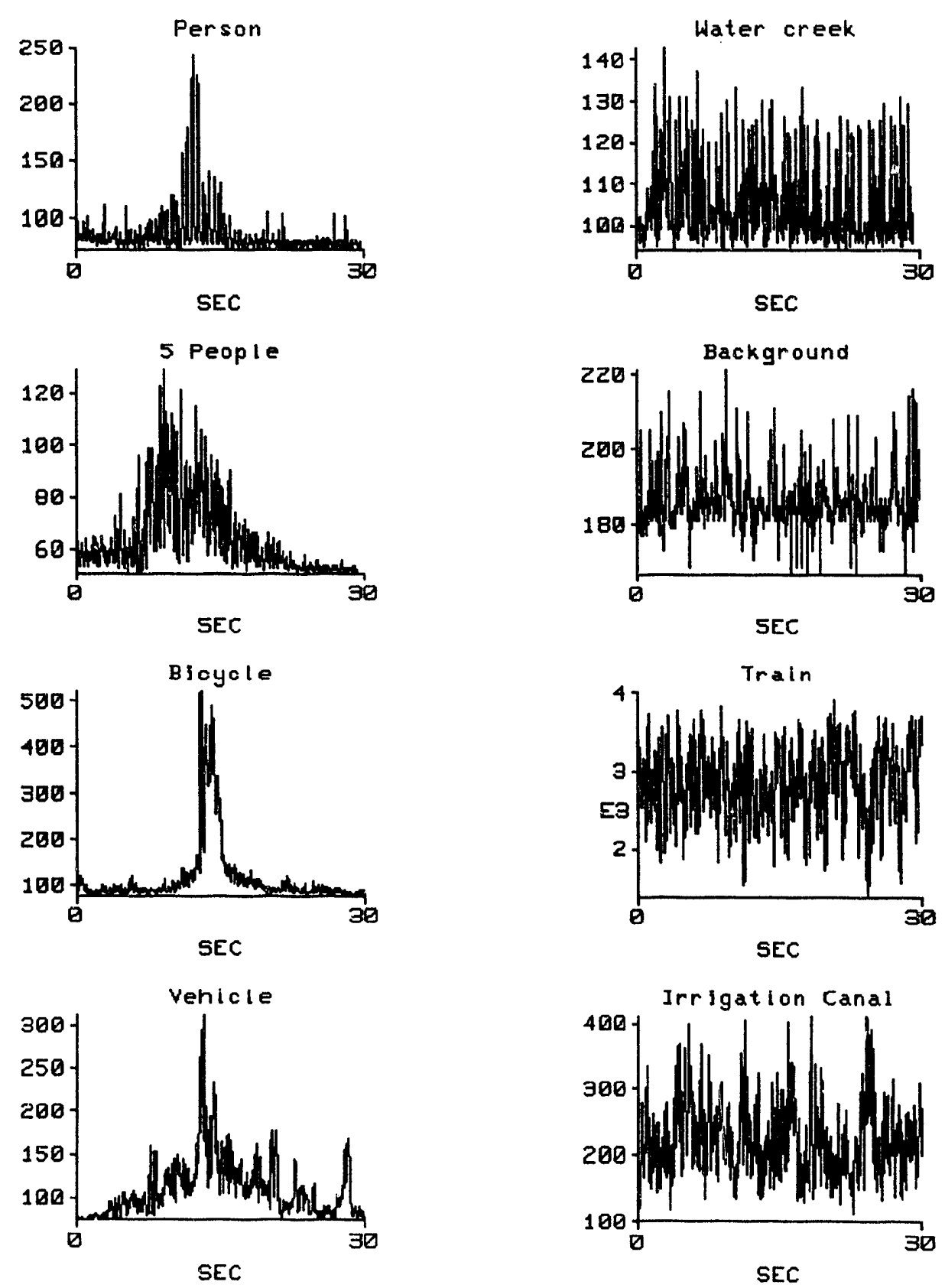

Figure 1: Example of seismic signals for the different categories.

\section{Event Detection Algorithm}

From Figure 1 it can be observed that one key feature for discriminating pedestrians and vehicles from the other events is the presence or absence of: a "bell-shaped" envelope in the waveform. In the case of pedestrians, or vehicles, the beil-shaped envelope reflects the fact that the pedestrian (or vehicle) approaches the sensor from a distance, and then it moves away, presumably at about the same speed. Although a train would also exhibit this kind of behavior, the time duration is typically much larger than an event caused by a pedestrian or vehicle, and therefore could be dis- 


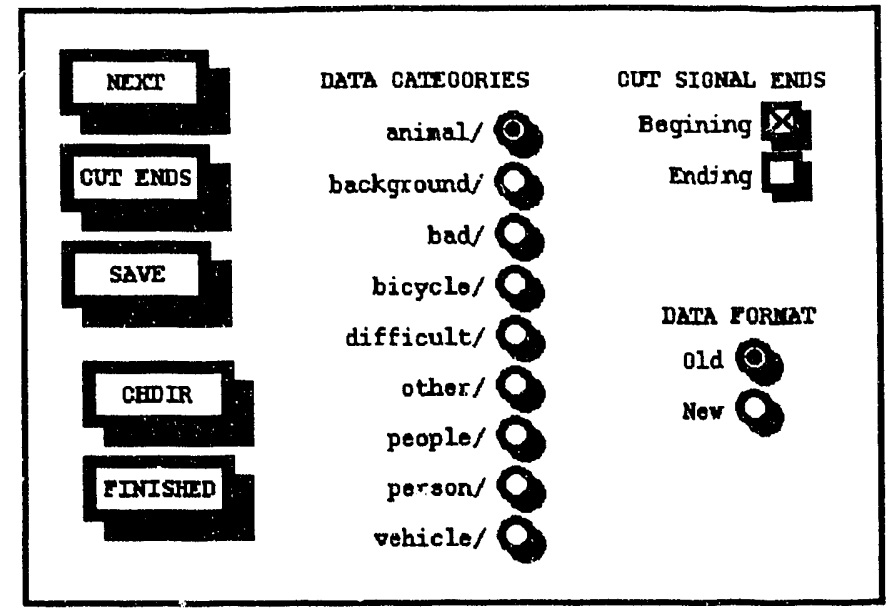

Figure 2: Graphical user interface tool for pre-screening and organizing the data.

criminated easily based on the time duration of the event. For example, most of our events lasted anywhere from 10 seconds to 30 seconds. A typical train would create a seismic signal that would last several minutes. This could also be true of other non-target events such as an irrigation canal or creek.

In order to quantify the presence and absence of a "bell-shaped" envelope we developed the follow ing algorithm. First we divide the waveform into three equal portions. Then the area under each of the three sections of the waveform is calculated. Finally, a ratio is calculated by dividing the averse area of the two conner sections by the area for the middle section. This ratio indirectly measures whether the event has a bell-shaped envelope or not. If the ratio is smaller than some predefined threshold value, then we assume that we have detected a target event. The algorithm is illustrated in Figure 3.

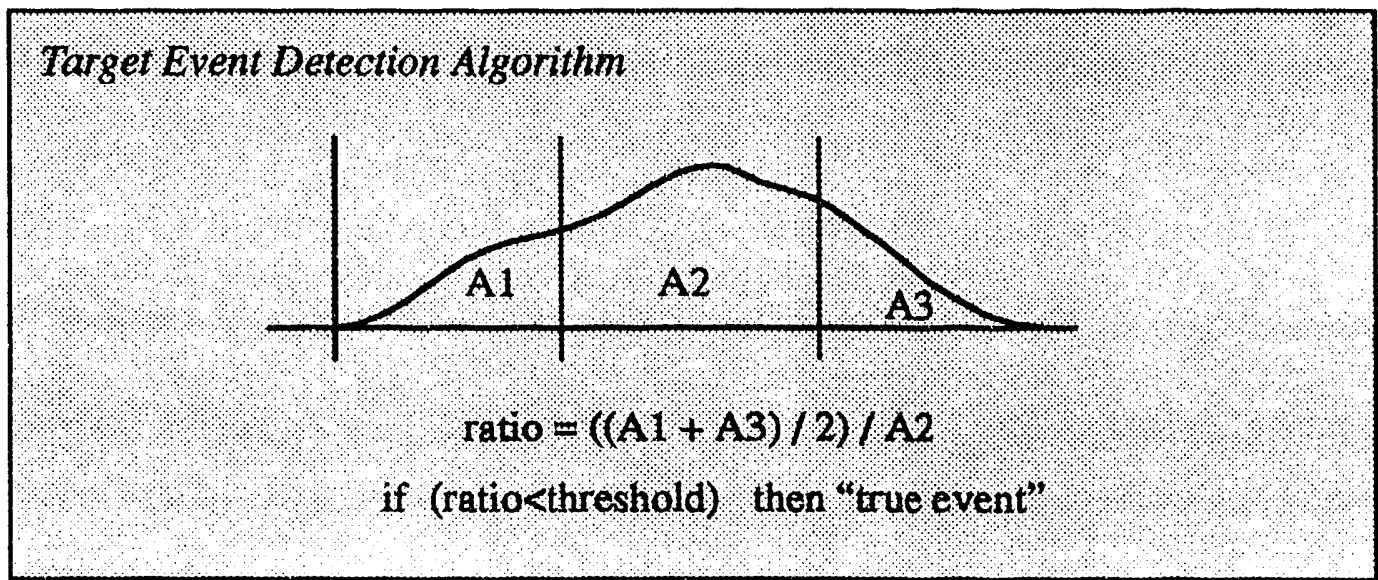

Figure 3: Algorithm for detecting a target event. 


\section{Pedestrian / Vehicle Classification Algorithm}

The current algorithm for classifying pedestrians and vehicles (see "Current Algorithm Overview" on page 2) has been improved. In the new algorithm we first remove the minimum value of the signal before calculating the ratio of the areas in order to compensate for the noise floor. This improves the classification performance of the algorithm for weak events.

We also improved the estimate of the area of the "pseudo-envelope"1. In the current algorithm the area of the envelope is calculated by integrating the values corresponding to the maximum value between the current waveform value and any previous value from $t=0$ to $t=i p e a k$, and $t=i$ last to $t=i p e a k$, where ipeak is the time corresponding to the peak value of the waveform and ilast is the time corresponding to the last value of the waveform. One disadvantage of this technique is that it can generate large biases on the estimate of the envelope area for vehicle events, which would result in low ratio values, typical of pedestrian events. This tends to happen due to irregularities in the ground, specially on dirt roads. In the new algorithm we use the local maximum extreme points of the waveform to form the envelope. Figure 4 shows some examples of the pseudo-envelope calculated for a vehicle and a pedestrian event using both the old and the new algorithms.

a)
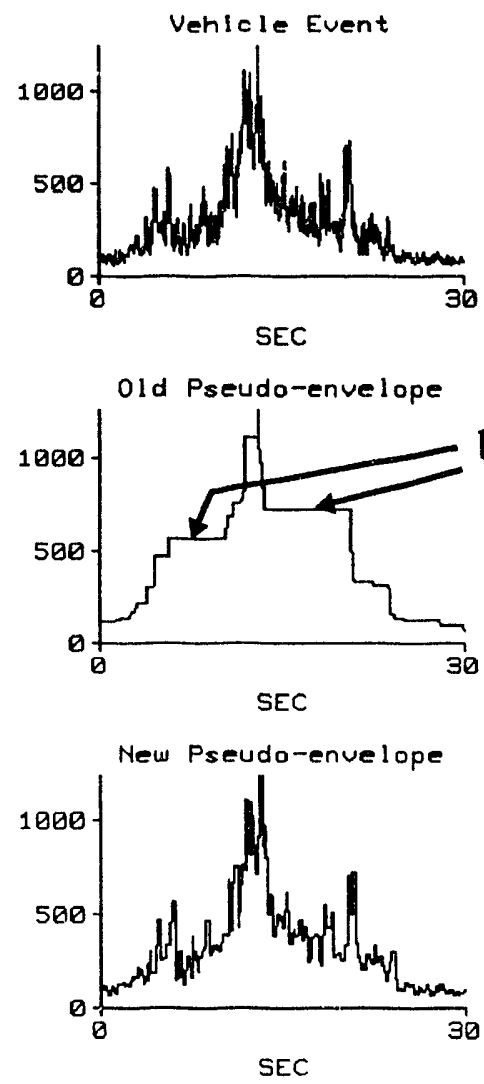

b)

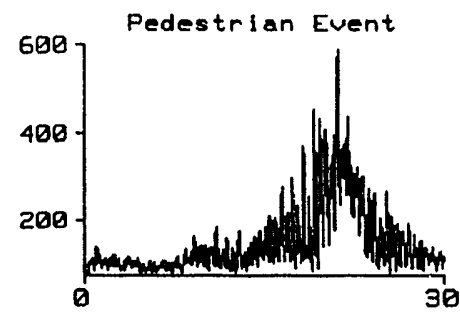

SEC
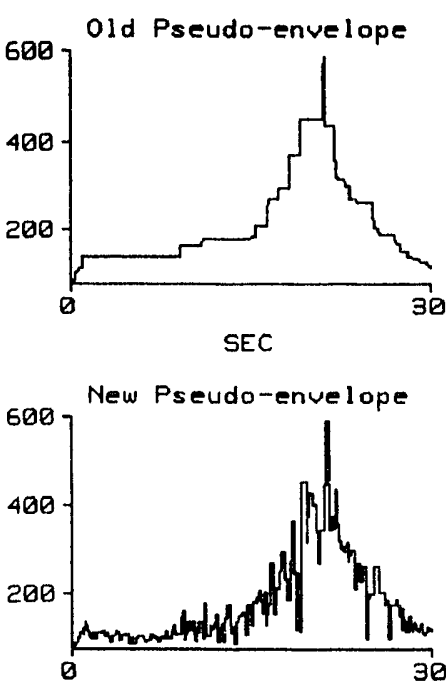

SEC

Figure 4: Seismic signatures and their pseudo-envelopes using the old and new algorithms for a a) vehicle, and b) pedestrian.

1. In order to minimize the power and processing requirements of the INSENS system, we do not calculate the true envelope of the signatures. 


\section{Algorithm Performance}

As described in the last two sections, our algorithm can classify an event as either vehicle, pedestrian, or other. In order to test the algorithm we had to simulate the triggering mechanism of the analog circuitry in the system since our data was collected manually. A scatter plot of the two features calculated for all the events in each category after simulating the triggering mechanism is shown in Figure 5. Note that for the most part it is possible to separate the three kinds of events by simply selecting the proper two threshold values.
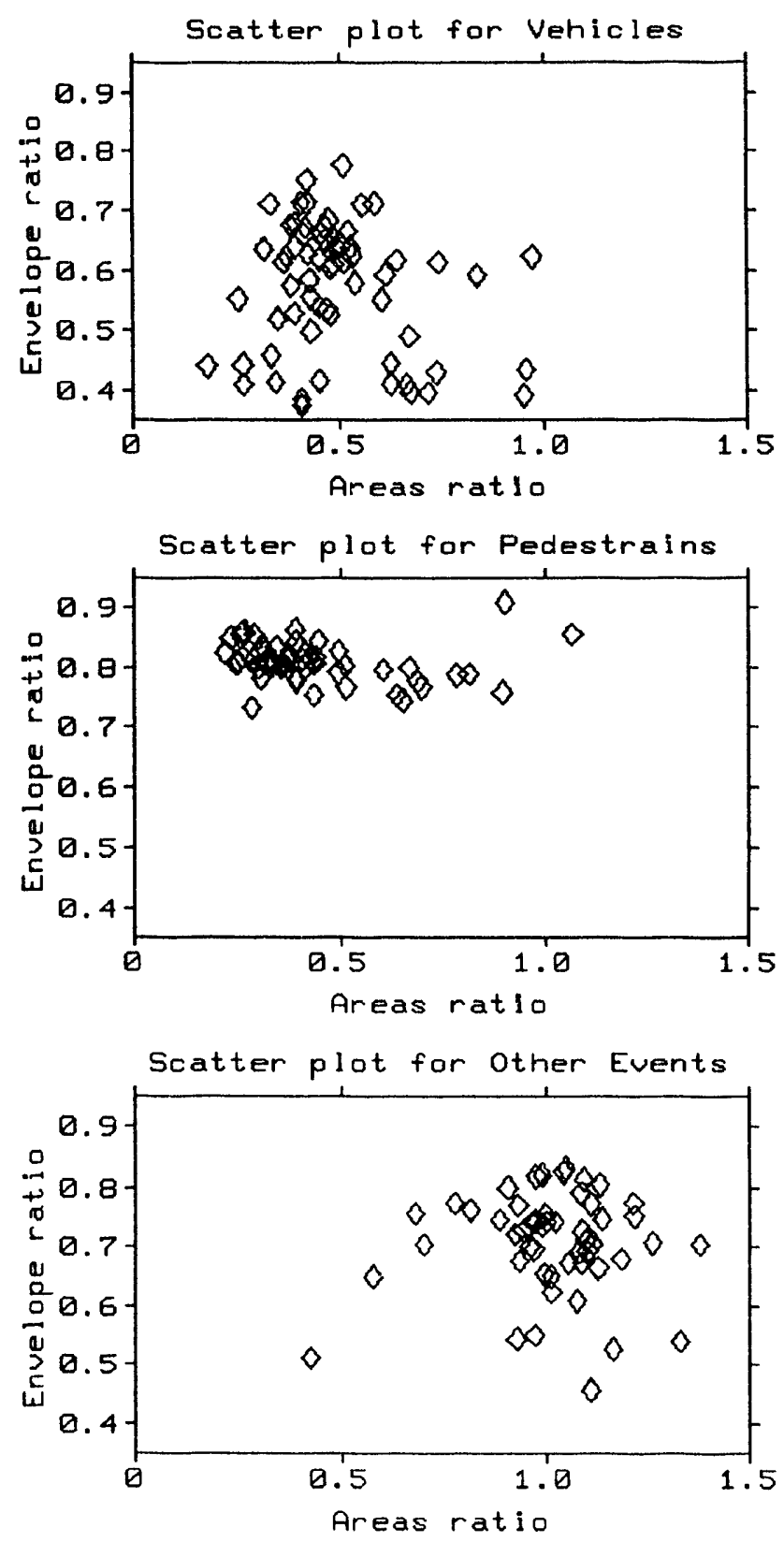

Figure 5: Scatter plot of features for the three types of events. 
Based on our data set we selected a threshold value of 0.85 for the areas ratio and a threshold value of 0.72 for the envelope ratio. Table 1 summarizes the classification results obtained for our current database using these threshold values.

\begin{tabular}{|c|c|c|c|}
\hline & vehicle & pedestrian & other \\
\hline \hline person & 0 & 22 & 4 \\
\hline people & 2 & 40 & 0 \\
\hline vehicle & 47 & 0 & 3 \\
\hline bicycle & 3 & 0 & 0 \\
\hline creek, canal, train & 3 & 2 & 19 \\
\hline background & 0 & 1 & 27 \\
\hline
\end{tabular}

Table 2: Classification results.

Based on these results we calculated the following probabilities for the new algorithm: the probability of detection for a vehicle is $94 \%$, the probability of detection for a pedestrian(s) is $91 \%$, the probability of missclassifying pedestrian and vehicle events is $2 \%$, the probability of missing a target event is $3 \%$, and the probability of nuisance alarm is $12 \%$.

We also simulated the current algorithm in the INSENS system for comparison purposes. A few minor modifications were made to the original algorithm in order to improve its performance.

Based on this simulation we obtained the following results: the probability of detection for a vehicle is $\mathbf{8 3 \%}$, the probability of detection for a pedestrian(s) is $91 \%$, the probability of missclassifying pedestrian and vehicle events is $12 \%$, the probability of missing a target event is $0 \%$, and the probability of nuisance alarm is $\mathbf{1 0 0 \%}$. The reason why the existing algorithm never misses an event is because it always classifies all events as pedestrians or vehicles. Unfortunately, this means that it will always classify all non-target events as target events.

\section{Other Work}

Other feature extraction techniques besides the ones described so far were also investigated. Nevertheless, the two features described in this report produced the most separable feature space.

Furthermore, due to the simple topology of the scatter plots for this data, a simple threshold-based classifier was used. It is possible as we increase the size of our data base and add other kinds of events that a more sophisticated algorithm such as a backpropagation neural network could provide better performance than the proposed algorithm. For completeness however, we will describe some of the other feature extraction techniques that were investigated.

The first feature extraction technique that was used consisted of calculating several central moments of the probability density function (pdf) of the data, the normalized histogram. Central moments can be used as features for representing shape information. In our case these features did not perform well since the shape of the pdf's were relatively complex, and not different enough for the different kinds of events. This particular set of features are known as histogram features and are define by the following equations, where $\mathrm{P}(\mathrm{x})$ represents the pdf of a waveform: 


$$
\begin{gathered}
\text { mean }=\bar{\mu}=\sum x \times P(x) \\
\text { sigma }=\sigma=\left[\sum(x-\mu)^{2} P(x)\right]^{1 / 2} \\
\text { skewness }=\frac{1}{\sigma^{3}} \sum(x-\mu)^{3} P(x) \\
\text { kurtosis }=\frac{1}{\sigma^{4}} \sum(x-\mu)^{4} P(x)-3 \\
\text { energy }=\sum[P(x)]^{2} \\
\text { entropy }=\sum P(x) \log [P(x)]
\end{gathered}
$$

We also attempted to use the central moments of the signatures directly to characterize the shape of the different events. These features were calculated using the same equations that were used for the pdf's, except that we replaced the pdf with a normalized version of the signal. These features did not work well either, mainly because they are not invariant to time scaling, i.e., they are sensitive to the absolute duration of an event; and in our case the duration of an event could vary substantially regardless of the type of event.

Another feature extraction technique that was tried consisted of generating pdf's for the sum and differences of neighboring values in the signal. Several features similar to the ones defined in Equations [1-6], were calculated from the resulting two pdf's. Some of these features generated more separable feature spaces than the ones described above. However, this technique is computationally expensive, since in general multiple pdf's need to be generated for different neighboring distances. This technique is commonly used for pattern recognition of textures.

\section{CONCLUSIONS}

A new algorithm was developed to detect and classify vehicles and pedestrians using seismic signatures collected with the INSENS geophone sensor. This algorithm is less sensitive to nuisance alarms than the previous algorithm. Furthermore, its computational requirements are not too demanding, and therefore suitable for implementation in a general purpose microprocessor such as the 8-bit microprocessor used in the INSENS system. We plan to install the new algorithm in the INSENS system and test and document its performance.

\section{COMMENTS}

There are still some kinds of nuisance events that have not been incorporateed into our database, such as animals, heavy rain, and tree vibrations due to wind. It is very likely that some animals will generate seismic signatures difficult to differentiate from pedestrians. It is also possible that tree vibrations due to gusty winds could create seismic signatures with a bell-shaped envelope, and therefore be classified as target events. If this proves to be the case, we will need to investigate other feature extraction techniques including Fourier analysis, as well as $m$ ore sophisticated classification algorithms such as neural networks. The feasibility of implementing these kinds of algorithms in the existing INSENS microprocessor may be an uissue. 
It should be noted that the proposed algorithm may not be adequate for applications requiring very low missclassifiation and nuisance alarm rates. One such scenario, which is of great interest to the border patrol, is the monitoring of roads with heavy vehicular traffic for the purpose of detecting pedestrians. This scenario would probably require false alarm rates on the order of 1 in 10,000 for it to be effective. We are currently proposing to attack this problem using data fusion techniques where several different transducers would be used together to classify an event. The details of this work will be available in a separate report in the near future. 


\section{Appendix A: Sources of Nuisance Alarms by Sensor and Location}

\begin{tabular}{|c|c|c|c|c|c|c|}
\hline & Categories & El Centro & El Paso & Washington & Vermont & Comments \\
\hline & tree roots & $\mathbf{x}$ & $\mathrm{x}$ & $\mathbf{x}$ & $\mathbf{x}$ & due to wind \\
\hline & wild animals & & $\mathbf{x}$ & $\mathbf{x}$ & $\mathbf{x}$ & deer, bears, etc. \\
\hline & cattle & & & & & \\
\hline & rodent & $\mathbf{x}$ & & & $\mathbf{x}$ & \\
\hline & train & $\mathbf{x}$ & $\mathbf{x}$ & $\mathbf{x}$ & $\mathbf{x}$ & \\
\hline & fence movement & $\mathbf{x}$ & $\mathbf{x}$ & & $\mathbf{x}$ & due to wind \\
\hline .0 & creeks & $\mathbf{x}$ & $\mathbf{x}$ & $\mathbf{x}$ & $\mathbf{x}$ & after rain \\
\hline 墨 & irrigation canals & $\mathbf{x}$ & $\mathbf{x}$ & & & \\
\hline & heavy rain & & $\mathbf{x}$ & & $\mathbf{x}$ & \\
\hline & water well pumps & $\mathbf{x}$ & $\mathbf{x}$ & & & \\
\hline & many people & & $\mathbf{x}$ & & & confused $w /$ vehicles \\
\hline & nearby vehicles & $\mathbf{x}$ & & & & \\
\hline & agric. vehicles & $\mathbf{x}$ & $\mathrm{x}$ & & $\mathbf{x}$ & \\
\hline & frozen ground & & & $\mathbf{x}$ & $\mathbf{x}$ & losses sensitivity \\
\hline & fence movement & & $x$ & & $\mathbf{x}$ & due to wind \\
\hline & track & & & & $\mathbf{x}$ & expands $w /$ heat \\
\hline$\frac{\Phi}{0}$ & train & $\mathbf{x}$ & $\mathbf{x}$ & & $\mathbf{x}$ & no response \\
\hline$\frac{\overline{8}}{0}$ & blowing sand & & $\mathbf{x}$ & & & carries minerals \\
\hline$\underset{\Xi}{\sigma^{0}}$ & creeks & & $\mathbf{x}$ & & & carries minerals \\
\hline & lightning & & $\mathbf{x}$ & $\mathbf{x}$ & $\mathbf{x}$ & \\
\hline & RF & $\mathbf{x}$ & $\mathbf{x}$ & $\mathbf{x}$ & $\mathrm{x}$ & local trans. \\
\hline & foliage movement & $\mathbf{x}$ & $\mathbf{x}$ & $\mathbf{x}$ & $\mathrm{x}$ & due to wind \\
\hline & background & $\mathbf{x}$ & $\mathbf{x}$ & $\mathbf{x}$ & $\mathbf{x}$ & due to clouds \\
\hline ఫ్రై & animals & $\mathbf{x}$ & & $\mathbf{x}$ & $\mathbf{x}$ & deer, bears, etc. \\
\hline & insects & $\mathbf{x}$ & & & $\mathbf{x}$ & spiders, etc. \\
\hline & hot days & $\mathbf{x}$ & & & & losses sensitivity \\
\hline
\end{tabular}



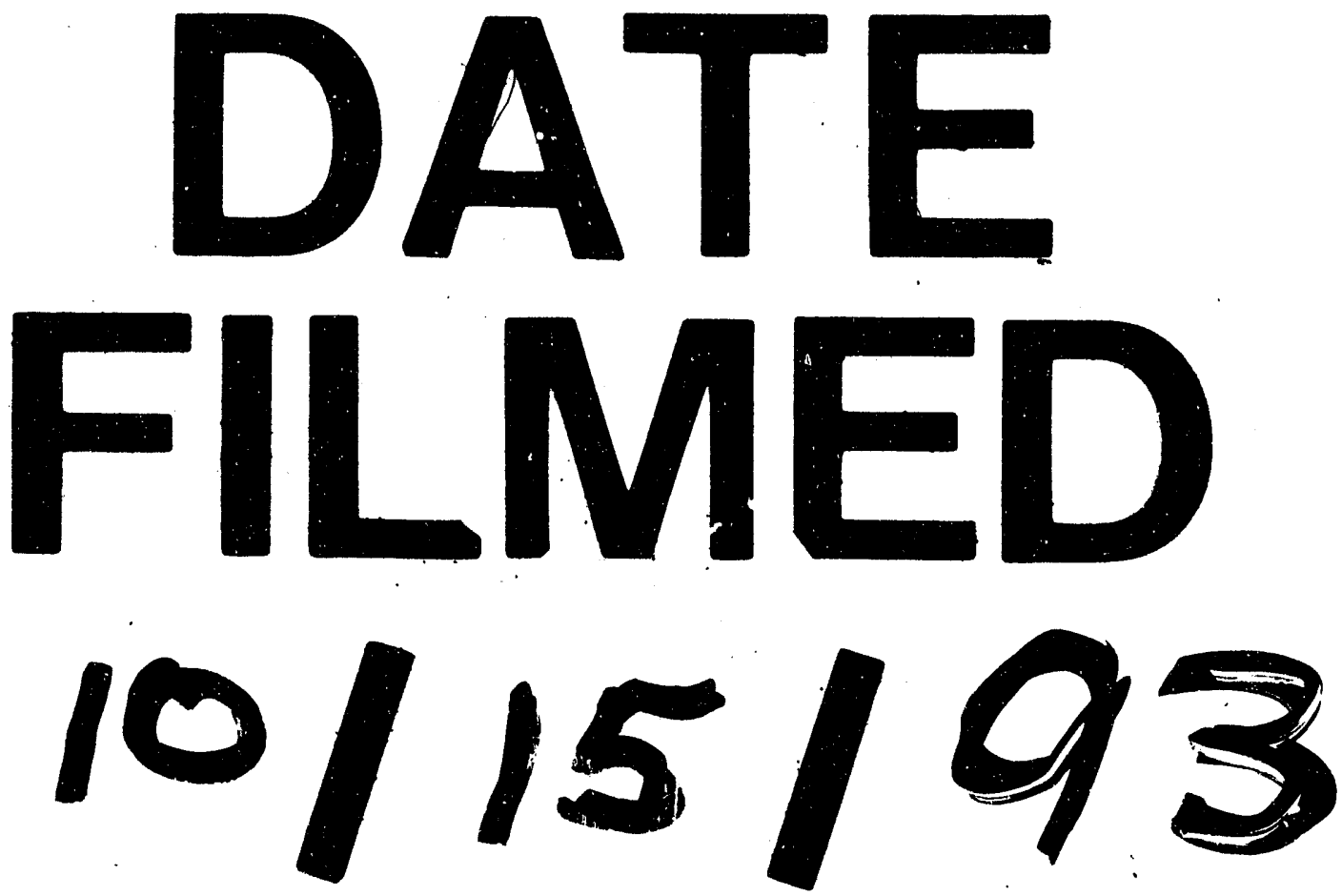

1 
